\section{Psicología IBEROAMERICANA}

\section{Psicología lberoamericana}

ISSN: 1405-0943

psicología.iberoamericana@uia.mx

Universidad Iberoamericana, Ciudad de México

México

Pinzón Luna, Bárbara Kristel; Pérez Villar, María de los Ángeles Estilos de apego y relación con la figura materna en mujeres que viven violencia conyugal

Psicología Iberoamericana, vol. 22, núm. 1, enero-junio, 2014, pp. 16-24

Universidad Iberoamericana, Ciudad de México

Distrito Federal, México

Disponible en: http://www.redalyc.org/articulo.oa?id=133944229003

- Cómo citar el artículo

Número completo

- Más información del artículo

Página de la revista en redalyc.org

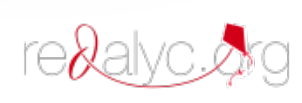

Sistema de Información Científica

Red de Revistas Científicas de América Latina, el Caribe, España y Portugal Proyecto académico sin fines de lucro, desarrollado bajo la iniciativa de acceso abierto 


\title{
Estilos de apego y relación con la figura materna en mujeres que viven violencia conyugal
}

\author{
Attachment style and relationship with the maternal figure among women \\ who experience domestic violence
}

\author{
Bárbara Kristel Pinzón Luna ${ }^{1}$ \\ Centro de Estudios e Investigación Gestálticos \\ María de los Ángeles Pérez Villar² \\ Universidad Popular de la Chontalpa
}

\section{RESUMEN}

La presente investigación consistió en conocer el estilo de apego de las mujeres que viven violencia conyugal, así como las características de la relación con la figura materna. La información se obtuvo mediante entrevistas a profundidad en cuatro mujeres que viven violencia conyugal, así como a las madres de cada una de ellas. Se encontró que: a) el estilo de apego que caracteriza a estas mujeres es el estilo ambivalente/preocupado; b) los modelos internos de trabajo están integrados por pensamientos como: ejerce poco control sobre su propia vida, demostraciones intensificadas para provocar respuestas en los demás; y c) las características de la relación con la figura materna encontradas son una respuesta adecuada a necesidades básicas, baja sensibilidad, abandono, poca contención emocional.

Palabras clave: teoría de apego, estilos de apego, violencia conyugal, modelos internos de trabajo, base segura.

\section{ABSTRACT}

This research examined the attachment styles of women who experience domestic violence, as well as the characteristics of the relationship with the maternal figure. The information was obtained through in-depth interviews with four women who experience domestic violence, as well as the mother of each of them. It was found that: a) these women typically show an ambivalent/anxious style of attachment; b) internal working models consist of thoughts such as: little control over their own life, intensified demonstrations to provoke responses from others; and c) the relationship characteristics with the mother figure are found to be an adequate meeting of basic needs, low sensitivity, abandonment, little emotional restraint.

Keywords: Attachment theory, attachment styles, marital violence, internal working models, secure base.

\footnotetext{
${ }^{1}$ Recibido: 9 de septiembre de 2013 - Aceptado: 4 de marzo de 2014.

Centro de Estudios e Investigación Gestálticos Cesigue. Estanzuela no. 10. Fracc. Pomona. C. P. 91040 Xalapa, Veracruz. Tel. (228) 817 51 99 y fax (228) 81835 88. www.cesigue.edu.mx

${ }^{2}$ Profesora investigadora de tiempo completo. Universidad Popular de la Chontalpa. Carretera Cárdenas-Huimanguillo Km 2 s/n Ranchería Paso y Playa. H. Cárdenas, Tabasco, C. P. 86500. Tel. 01 (937) 3727050 al 58, ext. 7005. www.upch.edu.mx
} 


\section{INTRODUCCIÓN}

En el ser humano existe una necesidad biológicamente determinada de la formación de vínculos de apego, que permitan la consecución de un sentimiento de seguridad psicológica (Bowlby, 1958). Dichos vínculos son determinantes en el desarrollo de la salud mental de una persona, debido a que ésta interpreta y maneja las relaciones posteriores con base en estas experiencias iniciales con el cuidador o la madre (Marrone, 2001). Una de las formas conocidas de vinculación se da a través de la violencia conyugal, la cual ocurre debido a una versión distorsionada de la conducta de apego desarrollada en la infancia.

Tomando la definición de apego expresada por Bowlby (1989): "la conducta de apego es cualquier forma de conducta que tiene como resultado el logro o la conservación de proximidad con otro individuo claramente identificado al que se considera mejor capacitado para enfrentarse al mundo". La teoría del apego es una explicación de la regulación del afecto, y como tal, diferencia a las personas en el modo en que éstas sienten y expresan sus necesidades de afecto, y en el modo en el que actúan para mantener un equilibrio emocional. Estos patrones de apego no son iguales en todos los individuos, sino que ante situaciones tales como protesta, desesperanza y separación hay una serie de reacciones y respuestas. Lo que permite una clasificación de estilos de apego, entre los que se describen: apego seguro, apego ambivalente/preocupado, apego evitativo y apego desorganizado (Ainsworth, Blehar, Waters \& Wall, 1978; Main \& Solomon, 1990).

$\mathrm{El}$ apego seguro posee un modelo mental de confianza en sí mismo y en los demás, que favorece el desarrollo de una mayor capacidad de afrontamiento y adaptación al entorno del niño, y en la etapa de adulto permite manejar el estrés ambiental de manera más eficaz que el resto de los estilos inseguros. Son personas abiertas, fáciles de conocer, con una alta autoestima y una imagen positiva tanto de sí mismos como de los demás, con mayor apertura cognitiva que el resto de los estilos, ausencia de problemas interpersonales graves, expectativa positiva de que los demás, generalmente, los aceptan y les responden (Hazan \& Shaver, 1987; Bartholomew, 1990; Feeney \& Noller, 1990; Bartholomew \& Horowitz, 1991; Collins \& Read,
1994; Feeney, Noller \& Callan, 1994; Mikuliner \& Daphna, 1999).

El apego ambivalente/preocupado se caracteriza, principalmente, por sentimientos de ambivalencia e inseguridad respecto a las figuras de apego. En sus relaciones adultas, se caracteriza por poseer una falta de valía personal que deriva en una actitud dependiente y un irascible deseo de conseguir la aprobación de los demás (Bartholomew, 1990; Bartholomew \& Horowitz, 1991). Desarrolla un estado de ansiedad y un sentimiento de ineficacia personal, no sintiéndose lo suficientemente querido, así como con falta de control sobre el ambiente; constantemente está en busca de una confirmación de que son queridos, y siempre muestra temor al posible abandono o rechazo (Melero, 2008).

En el apego evitativo, se ha aprendido a ocultar o controlar emociones tanto positivas como negativas. Posee un modelo mental positivo de sí mismo y negativo de los demás (Bartholomew, 1990; Bartholomew \& Horowitz, 1991). En cuanto a la relación establecida con sus padres, suelen ser experiencias de rechazo desprovistas de afecto, recuerdan a sus madres como frías y con una clara actitud de rechazo. Sus relaciones sociales se caracterizan por la distancia afectiva y la frialdad emocional, considerándolas como algo secundario a los aspectos profesionales o materiales (Feeney, Noller \& Callan, 1994; Mayseless, 1996); muestran una gran necesidad de éxito, perfeccionismo, adicción al trabajo o materialismo y sobreimplicación en tareas y actividades de logro (Brennan \& Shaver, 1995).

Por último, el apego desorganizado se caracteriza por una actuación parental estresante e incoherente para el niño, como situaciones de maltrato infantil, abuso, negligencias o la presencia de patologías en los cuidadores. Estas dinámicas propician la generación de modelos mentales en conflicto, puesto que la figura de apego es fuente de protección y de amenaza a la vez (Main \& Solomon, 1990).

Los estilos de apego descritos se diferencian en el tipo de estrategia utilizada para regular el estrés y los estados de inseguridad, o para minimizar su impacto. En este sentido, el porqué un estilo afectivo se mantiene estable a lo largo del tiempo va en relación con la funcionalidad y los beneficios que le reporta en el restablecimiento de su equilibrio emocional (Melero, 
2008). La estabilidad del apego a lo largo del desarrollo ha sido ampliamente investigada, obteniendo, en general, una estabilidad moderada-alta en la edad adulta (Collins \& Read, 1994; Feeney et al., 1994; Brennan \& Shaver, 1995; Waters, Weinfield \& Hamilton, 2000; Liebert \& Spiegel, 2000).

Esta estabilidad de los estilos de apego, puede asociarse con investigaciones que han encontrado que las consecuencias de los actos violentos tienden a repetirse en los hijos de las víctimas y se transmiten de generación en generación. Los vínculos de apego que los niños, víctimas de violencia intrafamiliar, establecen íntima y socialmente con otras personas son el punto de partida de la transmisión de patrones transgeneracionales de violencia (Amar \& Berdugo, 2006).

Melero (2008) señala que la estabilidad de los patrones de apego se debe principalmente a la resistencia al cambio de los modelos mentales de relación, que permanecen grabados en la memoria de las experiencias infantiles de relación y ejercen su influencia a través de las distintas circunstancias vitales por las que atraviesa el individuo. Algunos autores refieren que lo crítico para las relaciones íntimas de los adultos son los modelos mentales de los apegos, en principio porque los modelos mentales de uno mismo y de los demás son un componente central de la personalidad del sentimiento de identidad personal y del concepto de sí mismo; en segundo lugar, porque los modelos mentales están automatizados, por lo que una vez que son activados se desencadena una serie de conductas sobreaprendidas que operan fuera de la conciencia, y tercero, porque los modelos se autorrefuerzan, por lo que es el propio comportamiento y la actitud de la persona lo que provoca en los demás la respuesta que teme. Por otra parte, la inestabilidad en el estilo de apego se ha asociado a sucesos negativos que han supuesto un cambio consistente en el cuidado (Kirkpatrick \& Hazan, 1994; Hamilton, 2000; Waters et al., 2000).

La violencia conyugal es un fenómeno social que ocurre en un grupo familiar, y que consiste en el uso de medios instrumentales por parte del cónyuge o pareja, para intimidar psicológicamente o anular física, intelectual y moralmente a su pareja, con el objeto de disciplinar la vida familiar según su arbitrio y necesidad (Corsi, 1994). El Instituto Nacional de las Mujeres y el Instituto Nacional de Estadística y Geografía (2013) la definen como cualquier acción u omisión que cause a la pareja daño o sufrimiento psicológico, físico, patrimonial, económico o sexual, o bien, la muerte, tanto en el ámbito privado como en el público.

Una de las preguntas más frecuentes ante el fenómeno de la mujer maltratada es: ¿por qué ésta no rompe con el vínculo violento? Dentro de las explicaciones a esa permanencia junto al agresor, se encuentra el hecho de que muchas veces la víctima forma un vínculo afectivo con sus agresores, el cual va en aumento gradual, hasta que la mujer se identifica con su agresor, entendiendo y justificando el maltrato (Loubat, Ponce \& Salas, 2007).

Algunos estudios (Dutton, Starzomski, \& Van Ginkel, 1995; Dutton \& Golant, 1997; Magdol et al., 1998; Holtzworth-Munroe, Meehan, Herron, Rehman \& Stuart, 2003) apoyan la relación entre violencia con la pareja y el apego inseguro desarrollado en la infancia, y encuentran que un apego débil a los padres, caracterizado por poca calidez, confianza o comunicación positiva, exposición directa de violencia entre los padres, rechazo por parte de los padres, y un apego inseguro a las madres, predicen la situación de violencia en las parejas. Loubat, Ponce y Salas (2007), reportan que el tipo de apego tiene influencia en la actitud que presentan las mujeres ante la violencia conyugal; estaría caracterizado por factores como la ansiedad por separación -interpretada comoabandono-, que surge de sus experiencias infantiles al percibir a sus padres como no disponibles.

La violencia es un medio para recuperar el control sobre la figura de apego, y al menos, temporalmente, prevenir la amenaza de abandono de la pareja. La investigación relacionada con dicho planteamiento sugiere que la evitación al abandono y el control tiene origen en patrones de apego (Babcock, Jacobson, Gottman, \& Yerington, 2000; Waters et al., 2000).

En México, se estima que aproximadamente una tercera parte de las mujeres casadas o con una relación de unión libre ha vivido algún tipo de incidente violento relacionado con su pareja (INEGI, 2011). En Tabasco, la proporción de mujeres maltratadas a lo largo de su relación, es de las más altas en el país, al ocupar el quinto lugar, después de los estados de México, Jalisco, Colima y Durango (INEgi, 2011). De acuerdo con un informe elaborado por la Comisión Estatal de 
Derechos Humanos de Tabasco, A.C., la Secretaría de Salud de Tabasco (Gobierno del Estado de Tabasco, 2008) reportó que de enero de 2007 a mayo de 2009, se atendieron 3466 casos de violencia física, 4458 casos de violencia psicológica y 1067 casos de violencia sexual. Hasta el momento no se ha realizado en la entidad un estudio que permita profundizar en la comprensión de los modelos operativos internos de aquellas personas que viven agresión de sus parejas.

El propósito de la presente investigación consistió en conocer las características de la relación con la figura materna, así como el estilo de apego de las mujeres que viven violencia conyugal. Las principales preguntas a responder fueron: ¿cuáles son los estilos de apego de las mujeres que viven violencia conyugal?, y ¿cuáles son las características de los vínculos de apego de la mujer que vive violencia conyugal?

\section{MÉTODO}

\section{Participantes}

Este estudio se efectuó según la perspectiva metodológica cualitativa, con el apoyo de entrevistas a profundidad. Se conformó una muestra de cuatro mujeres que acuden voluntariamente a un consultorio de Psicología en el estado de Tabasco, de clase socioeconómica media, con al menos cinco y hasta 14 años de matrimonio, con un promedio de dos hijos por pareja y que viven violencia conyugal. Las edades de las participantes oscilaron entre los 30 y 45 años. Asimismo, se entrevistó a las madres de cada una de las participantes, cuyo rango de edad se encontró entre los 56 y 69 años. En todos los casos se buscó un consentimiento informado y se trabajó en condiciones de privacidad y confidencialidad.

\section{Instrumentos}

Se utilizó una adaptación de la entrevista basada en la modalidad terapéutica denominada Intervención breve basada en el apego о ваві (por sus siglas en inglés: Brief Attachment Behavior Intervention), desarrollada por Jeremy Holmes (2009), la cual proporciona estímulo para la construcción narrativa al hacer preguntas diseñadas para activar el recuerdo de experiencias relacionadas con el apego en las entrevistadas. En las palabras de Fonagy (2004), el estilo narrativo o función reflexiva puede ser una guía para el diagnóstico, al señalar los patrones de relación, en particular en la infancia, y tendencias en la vida adulta. Aborda los siguientes dominios de la teoría de apego: base segura, pérdida, protesta, sana-ira y asertividad, modelos internos de trabajo y función reflexiva. Cada dominio está conformado por una serie de preguntas divididas en dos tiempos, las relacionadas con la infancia y las relativas a la conducta actual.

Respecto a las madres de cada una de estas mujeres, se diseñó una entrevista con preguntas abiertas que tuvo como propósito examinar las características de los vínculos afectivos establecidos en la infancia y de la dinámica familiar de las participantes, con base en los dominios de la entrevista вABI (Holmes, 2009).

\section{Procedimiento}

Todas las entrevistas se grabaron a lo largo de dos años para su posterior transcripción y categorización. La duración total de estos encuentros fue aproximadamente de dos horas, de acuerdo con el ritmo de los entrevistados y sus características personales. La mayoría de ellas se realizó en un consultorio de psicología, salvo dos que se efectuaron en el domicilio de la persona, por motivos de edad. Por estas razones, cada una se llevó a cabo en dos o tres encuentros.

Una vez efectuadas las entrevistas, se transcribieron y se procedió a examinar los datos, conservando su naturaleza textual e identificando los patrones de significantes para obtener una descripción densa y sólida de los datos. Posteriormente, se examinó el significado de frases o párrafos específicos, para luego codificar y categorizar las unidades de acuerdo con determinados componentes temáticos. Al tomar en consideración las aproximaciones teóricas y estrategias metodológicas en que se fundamenta esta investigación, se optó por presentar descripciones detalladas, intercaladas con párrafos de análisis, utilizando un lenguaje claro y directo para favorecer la comprensión de lo redactado.

\section{RESULTADOS}

En general, se puede mencionar que el estilo de apego que caracteriza a las mujeres entrevistadas es el ambivalente/preocupado. Respecto a los dominios de BABI, se encontró en la categoría de base segura que 
tres de las mujeres de esta investigación recurrían a sus "madres" como principales fuentes de seguridad. De acuerdo con los relatos, estas figuras que funcionaron como base segura se caracterizaron por una respuesta ambivalente e impredecible, baja disponibilidad, baja implicación emocional; una respuesta adecuada a las necesidades físicas y de protección, pero no sustancial ante las demandas afectivas:

"Hubo un antes y un después de que mi mamá se fuera. No comprendía lo que esto significaba”.

"Mi mamá vivía muy presionada, trabajando mucho, lo que hizo que su carácter cambiara un poco; nos gritaba todo el tiempo y se enojaba con facilidad".

"Sí, recuerdo que había momentos en que no era mi mamá, sino mis hermanas las que se encargaban de mí".

"Era poco cariñosa, no daba muchos abrazos y la mayor parte del tiempo estaba haciendo algo de la casa; no jugó conmigo ni con mis hermanos ni hermanas; como que sólo se preocupaba por la comida y por las cosas de la escuela".

“...Luisa no lloraba cuando me iba ni se enojaba, aunque no creo que me fuese a extrañar mucho. A diferencia de con su padre, a él sí salía a recibirlo...”

Las características anteriores pueden sugerir una experiencia de sensación de abandono, soledad e impotencia, lo que puede provocar una intensa ansiedad y por ello podría no desarrollarse la suficiente confianza en sí mismo. Cabe mencionar que una de ellas vive abandono por parte de la madre, lo que la lleva a buscar seguridad en la abuela paterna.

En cuanto a la categoría de protesta, en las mujeres entrevistadas podemos observar que en su mayoría prevalece la tendencia a ceder, con expresión de la protesta, incluso con la presencia de estallidos ante esta misma, pero sin la resolución asertiva de la emoción, por llegar a considerarlo poco importante para otros. “...solía quedarse callada, por ejemplo cuando la regañaba, no contestaba, ni de mayorcita tampoco lo hacía cuando sus hermanos le hacían travesuras..."

“...cuando la regañaba, sí lloraba pero sin reclamar. Tampoco fue una niña que pidiera mucho porque pues no había mucho y aceptaba lo que se le daba..."

La categoría que se refiere a la pérdida, es en gran parte la angustia psicológica. La capacidad para afrontar la pérdida es un componente clave de la madurez psicológica. Es decir, tendrá la capacidad de acercarse al mundo con seguridad, pero en caso de dificultades estará dispuesta a buscar apoyo en figuras de confianza. Por lo que cuando la madurez no se alcanza, se relaciona con lazos afectivos arcaicos difíciles de romper, dependencias, miedos, debilidad del yo que prefiere vivir como una prolongación de otro. $\mathrm{Y}$ en las relaciones adultas, cada miembro trae consigo un historial de separaciones y pérdidas y de una base interna que influirá en el estilo de relación (Holmes, 2009). Las mujeres en esta investigación experimentan angustia, miedo, desconcierto, tristeza, llanto y frustración ante la amenaza de separación.

“...la muerte de mi papá fue y sigue siendo la pérdida más grande que he vivido hasta ahora. En la casa todo se modificó por completo. Fue sumamente duro para mí que me despedí de él y de pronto ya no regresó. Mi mayor soporte se me fue. Me sigue haciendo mucha falta..."

"...ante situaciones difíciles desearía que estuviera aquí para darme seguridad y amor...”

Los modelos internos de trabajo son aquellos que se establecen durante los primeros años de vida y consisten en las formas en que se ejecutan las cosas, así como las conductas self-otros que influyen en la constitución de un ser humano (Holmes, 2009). Los modelos internos de trabajo de las mujeres entrevistadas están integrados por pensamientos como: ejerce poco control sobre su propia vida, no sabe reaccionar ante situaciones de tensión, deseo de extrema intimidad, demuestra intensificadamente para provocar respuestas en los demás y ser solícitos. En cuanto a la función reflexiva-la cual es la 
habilidad para pensar en las propias experiencias, con coherencia y cohesión sobre el sí mismo y los demás-, puede encontrarse coherencia en el discurso y equilibrio entre recuerdos placenteros y no placenteros.

Lo anterior indica signos de inseguridad respecto a la accesibilidad de la figura de apego, miedo al abandono y ansiedad de rechazo. Hay conductas de dependencia y temprana apertura emocional, necesidad de contacto y atención, sobreimplicación, deseo de un compromiso exclusivo, pero dificultan este proceso debido a sus constantes demandas basadas en una insatisfacción personal. Respecto a la figura materna, ésta se caracterizó por una adecuada percepción y respuesta a necesidades básicas, infraestimulación, abandono, baja disponibilidad y sensibilidad (tabla 1).

\section{DISCUSIÓN}

La teoría del apego proporciona una manera de entender cómo los seres humanos desarrollan fuertes lazos con los demás (Bowlby, 1979). A través de la interacción del niño con los padres, los menores desarrollan modelos internos de trabajo de uno mismo, que se caracterizan por el grado de dependencia emocional de otras personas para autovaloración, y los modelos internos de trabajo de otros, que se caracterizan por las expectativas acerca de la disponibilidad de los demás (Bowlby, 1973).
La perspectiva del apego en el amor romántico sugiere que las relaciones de pareja son, primariamente, relaciones de apego, cuyas funciones son transferidas a la pareja siguiendo procesos similares a los experimentados durante la infancia (Hazan \& Shaver, 1987). Esto no quiere decir que los individuos tendrán el mismo estilo de apego con su pareja en la vida adulta como el que tuvieron con sus padres; sin embargo, sus relaciones tienen la misma base de seguridad que se buscó en la infancia.

Con base en lo observado en este trabajo, es posible advertir que las mujeres evaluadas presentan un estilo de apego ansioso/ambivalente. La persona con este estilo de apego desarrolla un estado de ansiedad y un sentimiento de ineficacia personal, no sintiéndose lo suficientemente querida, así como una sensación de falta de control sobre el ambiente, como afirma Melero (2008). La constante insatisfacción, tanto real como derivada de su modelo mental, que asume que sus actos no tienen relación directa con sus consecuencias, las convierte en personas que no sólo están buscando la constante confirmación de que son queridas, sino que además creen ser ineficaces socialmente e incapaces de hacerse querer de modo estable, mostrando siempre un temor al posible abandono o rechazo.

Respecto a las características de los estilos de apego establecidos en la infancia de las mujeres entrevista-

Tabla 1. Características de apego de las mujeres y de la relación con la figura materna

\begin{tabular}{l|l}
\hline Mujeres & \multicolumn{2}{l}{\begin{tabular}{l}
\multicolumn{1}{l}{ Figura materna } \\
\hline $\begin{array}{l}\text { Inseguridad respecto a la accesibilidad de la figura } \\
\text { de apego y la propia valía }\end{array}$
\end{tabular}} & $\begin{array}{l}\text { Percepción, interpretación y respuesta } \\
\text { adecuada a necesidades básicas }\end{array}$ \\
\hline Miedo al abandono & Sensación de injusticia \\
\hline Ansiedad de rechazo & Infraestimulación \\
\hline Necesidad de contacto y atención & Abandono \\
\hline Sobreimplicación e idealización & Baja disponibilidad \\
\hline Sentimiento de no sentirse amada y/o agradable & Baja implicación conductual \\
\hline Excesiva y temprana apertura emocional & Poca contención emocional \\
\hline Necesidad de compromiso excesivo & Baja sensibilidad \\
\hline
\end{tabular}


das que viven violencia conyugal, se observa que mantuvieron una interacción con la figura materna que se caracteriza por una respuesta adecuada a las necesidades básicas; sin embargo, las necesidades afectivas no fueron del todo percibidas como satisfactorias y complementarias. Es decir, la figura materna se caracterizó por ser inconsistentemente responsiva, con baja disponibilidad, indiferencia, baja implicación conductual, infraestimulación, inaccesibilidad e inconsistencia concurrente.

Si las figuras parentales son incapaces de ayudar a sus hijos cuando éstos manifiestan temor, se desarrolla en la infancia una excesiva sensibilidad frente a cualquier estrés, el cual se expresará en la vida adulta como una incapacidad para hacer frente a cualquier situación conflictiva. Cuando la respuesta es cambiante e impredecible, tanto en intensidad como en contenido emocional, genera en el niño una falta de sentido de lo que está pasando y de control sobre su entorno. Los niños no logran obtener suficiente confianza en sí mismos. Lamentablemente, esta incoherencia entre lo que el niño hace y la respuesta de la madre influirá de manera negativa en el desarrollo de los procesos cognitivos, sobre todo, en los aspectos relacionados con el terreno social y afectivo. El sentimiento constante de no sentirse lo suficientemente amado(a), y agradable para el otro, influye en forma negativa en su autoestima, autoconcepto y en la visión del mundo. Lo anterior se ha encontrado en la literatura, es una de las conductas que permite el desarrollo de un estilo de apego ambivalente/preocupado (Schore, 1994).

Parecen ser los factores que hacen que estas mujeres tengan el sentimiento constante de no sentirse lo suficientemente amadas o agradables para los otros, que puedan considerar la violencia ejercida por sus parejas como algo natural y que esperan poco o nada del amor o el apoyo de alguien. Como lo mencionan Main, Kaplan y Cassidy (1985), los padres transmiten a sus hijos modelos internos de apego, cuya influencia se dejará ver, principalmente, en el tipo de relaciones íntimas que se establecerán en el futuro con sus parejas.

Holmes (2009) menciona que el sujeto buscará a una pareja que pueda tolerar el patrón dictado por sus modelos internos de trabajo, pero también cada uno de ellos será infeliz con las restricciones que esto conlleva; por tanto, toda relación contiene también la espe- ranza de trascender los viejos patrones. Sin embargo, podría decirse que las personas que han adoptado estrategias inseguras, basadas en experiencias con cuidadores inaccesibles o inconsistentes, tienden a formar relaciones donde no se sienten apoyados y serán fácilmente perturbables.

En los casos mencionados, en efecto, existió contención emocional en determinados momentos, pero aquellos que influyeron en la conformación de los sentimientos de valía no fueron suficientemente sólidos para desarrollar una imagen positiva del sí mismo. Como lo menciona Shore (1994), los cuidadores que son capaces de generar estimulación en el menor, pero no son del todo sensibles al momento de reducirla, interfieren en la capacidad del niño para autorregularse con posterioridad.

El marco conceptual del apego sostiene que las diferencias en las tendencias del amor de pareja tienen su origen en las experiencias tempranas con los cuidadores, y los procesos mediadores que implican modelos mentales del apego pueden explicar tanto la continuidad como la posibilidad de cambio en los patrones relacionales tempranos. De este modo, no ve el amor de pareja como un fenómeno aislado, sino como una parte integrante del vínculo afectivo humano. También la teoría es lo suficientemente amplia para englobar una serie de aspectos relacionales como el amor, la ansiedad, la soledad y la pérdida. Y, por último, la perspectiva del apego permite explicar formas sanas y disfuncionales de amor utilizando los mismos principios generales; se cree que las diversas formas de amor se originan como adaptaciones predecibles a circunstancias específicas.

Si bien la violencia conyugal se ha estudiado y abordado desde diversos enfoques, la teoría del apego puede proporcionar y servir como una guía de intervención psicoterapéutica en estos casos. Permite la evaluación de los vínculos afectivos, a través de su historia personal, sobre todo en lo referente a separaciones y encuentros con otros (por eventos sociales, educativos, enfermedades, fallecimientos, etc.), así cómo la reacción ante ellos; se presta especial atención a las discrepancias entre la descripción de los hechos y las personas (respecto a eventos pasados) y sus recuerdos de estas situaciones. También permite evaluar el patrón de comportamiento de apoyo del 
paciente a partir de lo que refiere sobre cómo se relacionó y se relaciona con otros y consigo mismo. Arietta Slade (1999), menciona que la teoría del apego es relevante para la práctica clínica: "En esencia, las categorías de apego cuentan una historia. Una historia sobre cómo la emoción se ha regulado, qué experiencias ha permitido la conciencia, y en qué medida una persona ha sido capaz de hacer sentido de su o sus relaciones primarias".

En el caso del estado de Tabasco, las organizaciones que se dedican a la prevención y tratamiento de la violencia conyugal utilizan el enfoque conductual, que se basa en la idea de que la mayor parte de la conducta (incluida la inadaptada) es aprendida, centrándose en la modificación de las conductas observables inadecuadas. Por lo que el abordaje clínico desde la perspectiva de la teoría del apego puede permitir el desarrollo de herramientas que apunten a la transformación de la base segura, trabajando la reparación de los vínculos infantiles; la imagen de sí mismas y de los otros signi-

\section{REFERENCIAS}

Ainsworth, M. D., Blehar, M. C., Waters, E. \& Wall, S. (1978). Patterns of attachment: a psychological stu$d y$ of the strange situation. New Jersey: Lawrence Erlbaum Associates.

Amar, J.A. \& Berdugo, M. (2006). Vínculos de apego en niños víctima de la violencia intrafamiliar. Psicología desde el Caribe, 18, 1-22.

Babcock, J. C., Jacobson, N. S., Gottman, J. M. \& Yerington, T. P. (2000). Attachment, emotional regulation and the function of marital violence: differences between secure, preoccupied, and dismissing violent and non-violent husbands. Journal of Family Violence, 15(4), 391-409.

Bartholomew, K. (1990). Avoidance of intimicy: an attachment perspective. Journal of Social and Personal Relationships, 7, 147-178.

Bartholomew, K. \& Horowitz, L. M. (1991). Attachment styles among young adults: a test of a four-category model. Journal of Personality and Social Psychology, 61, 226-244.

Bowlby, J. (1958). The nature of the child's tie to his mother. International Journal of Psychoanalysis, 39, 350373. ficativos; formas de enfrentar pérdidas y temores, además de desarrollar habilidades más eficaces para la resolución de conflictos.

Una primera limitación de este trabajo fue considerar únicamente a la figura materna como el vínculo primario. En efecto, la relación con ella tuvo influencia en el desarrollo psicoemocional de los participantes, pero también pudo observarse la participación de otras figuras de apego importantes, tales como el padre o los abuelos, quienes resultaron cruciales en el desarrollo de las participantes. Una segunda limitación fue no considerar las características de vinculación con la figura materna de las parejas que ejercen violencia. Por lo que para siguientes investigaciones, se sugiere contemplar dichas entrevistas para ahondar en el tema y lograr un mayor conocimiento acerca de la influencia de los vínculos afectivos generados durante la infancia y la vida adulta, así como su relación con la violencia conyugal.

Bowlby, J. (1973). Attachment and loss: separation, anxiety and anger. New York: Basic Books.

Bowlby, J. (1979). The making and breaking of affectional bonds. London: Tavistock.

Bowlby, J. (1989). Una base segura. Aplicaciones clínicas de una teoría del apego. Buenos Aires: Paidós.

Brennan, K.A. \& Shaver, P.R. (1995). Dimensions of adult attachment, affect regulation and romantic relationship functioning. Personality and Social Psychology Bulletin, 21, 267-283.

Collins, N. R. \& Read, S. J. (1994). Cognitive representations of attachment: the structure and function of working models. En K. Bartholomew y D. Perlman (Eds.), Advances in personal relationships (pp. 53-90). London: Jessica Kingsley Publishers.

Corsi, J. (1994). Una mirada interdisciplinaria sobre un grave problema social. Buenos Aires: Paidós.

Dutton, D.G., Starzomski, A. \& Van Ginkel, C. (1995). The role of shame and guilt in the intergenerational transmission of abusiveness. Violence and victims, 10(2), 121-131.

Dutton, D. \& Golant, S. (1997). El golpeador: perfil psicológico. Buenos Aires: Paidós. 
Feeney, J. A. \& Noller, P. (1990). Attachment style as a predictor of adult romantic relationships. Journal of Personality and Social Psychology, 58, 281-291.

Feeney, J. A., Noller, P. \& Callan, V. J. (1994). Attachment style, communication and satisfaction in the early years of marriage. En D. Perlman y K. Bartholomew (Eds.), Advances in Personal Relationships (pp. 269308). London: Jessica Kingsley.

Fonagy, P. (2004). Teoría del apego y psicoanálisis. Barcelona: Espaxs.

Gobierno del Estado de Tabasco. Comisión Nacional de Derechos Humanos de Tabasco, A.C. (2008). Panorama de violencia contra las mujeres. Tabasco, México: Comisión Nacional de Derechos Humanos de Tabasco, A.C.

Hamilton, C. E. (2000). Continuity and discontinuity of attachment from infancy through adolescence. Child Development, 71, 690-694.

Hazan, C. \& Shaver, P.R. (1987). Conceptualizing romantic love as an attachment process. Journal of Personality and Social Psychology, 52, 511-524.

Holmes, J. (2009). Teoría del apego y psicoterapia. En busca de la base segura. Bilbao: Descleé de Brower.

Holtzworth-Munroe, A., Meehan, J.C., Herron, K., Rehman, U. \& Stuart, G.L. (2003). Do subtypes of maritally violent men continue to differ over time? Journal of Consulting and Clinical Psychology, 71, 728-740.

Instituto Nacional de Estadística y Geografía (2011). Censo de Población y Vivienda 2010. Estados Unidos Mexicanos. Resultados preliminares. México: INEGI.

Instituto Nacional de las Mujeres e Instituto Nacional de Estadística y Geografía (2013). Mujeres y hombres en México 2012. México: Inmujeres/INEGI.

Kirkpatrick, L. A. \& Hazan, C. (1994). Attachment styles and close relationships: a four-year prospective study. Personal Relationships, 1, 123-142.

Liebert, R. M. \& Spiegel, L. L. (2000). Personalidad: estrategias y temas. México: Thomson Editores.

Loubat, M; Ponce, P. \& Salas, P. (2007). Estilo de apego en mujeres y su relación con el fenómeno del maltrato conyugal. Terapia Psicológica, 25(2), 113-122.

Magdol, D., Moffitt, T., Caspi, A., Newman, D., Fagan, J. \& Silva, P. (1998). Gender differences in partner vio- lence in a birth cohort of 21 years old: bridging the gap between clinical and epidemiology approaches. Journal of Consulting and Clinical Psychology, 65, 68-78.

Main, M., Kaplan, N. \& Cassidy, J. (1985). Security in infancy, childhood and adulthood: a move to the level of representation. En I. Bretherton y E. Waters (Eds.), Growing points of attachment theory and research. Monographs of the Society for Research in Child Development, 50, 66-104.

Main, M. \& Solomon, J. (1990). Procedures for identifying infants as disorganized/ disoriented during the ainsworth strange situation. En M. T. Greenberg, D. Cicchetti y E. M. Cummings (Eds.), Attachment in the preschool years. Theory, research and intervention (pp. 121-160). Chicago: The University of Chicago Press.

Marrone, M. (2001). La teoría del apego. Un enfoque actual. Madrid: Psimática.

Mayseless, O. (1996). Attachment patterns and their outcomes. Human Development, 39, 206-223.

Melero, C.R. (2008). La relación de pareja. Apego, dinámicas de interacción y actitudes amorosas: consecuencias sobre la calidad de la relación. Valencia: Universitat de València. Servei de Publicacions.

Mikuliner, M. \& Daphna, A. (1999). Attachment working models and cognitive openness in close relationships: a test of chronic and temporary accessibility effects. Journal of Personality and Social Psychology, 77, 710-725.

Schore, A. N. (1994). Affect regulation and the origin of the self: the neurobiology of emotional development. New Jersey: Lawrence Erlbaum Associates.

Slade, A. (1999). Attachment theory and research: implications for the theory and practice of individual psychotherapy. En J. Cassidy \& P. R. Shaver (Eds.). Handbook of Attachment: Theory, Research, and Clinical Applications (pp. 575-594). New York: Guilford.

Waters, E., Weinfield, N. S. \& Hamilton, C. E. (2000). The stability of attachment security from infancy to adolescence and early adulthood: general discussion. Child Development, 71, 703-706. 